\section{Diffraction Tomographic Imaging with a Circular Array}

Prabhakar S. Naidu, A. Vasuki, P. Satyamurthy, and L. Anand

Abstract-A new approach of imaging an object profile from the scattered wavefield, which is measured using a large (far field approximation) circular array of sensors surrounding the object, is described. It is shown that the measured field is itself proportional to the Fourier transform of the object. Consequently, as compared to the existing methods, such as back propagation method for a linear array, our method overcomes the well known effects of the finite window in numerical evaluation of the Fourier transform of the measured scattered field. This results into improved reconstruction.

\section{INTRODUCTION}

Diffraction tomography refers to cross-sectional imaging of objects from diffracted or scattered wavefield. (A good review of diffraction tomography may be found in [1]-[2]). An object is illuminated from various directions with a diffracting source of energy such as acoustic waves whose wavelength is comparable with the scale of inhomogeneities. The incident wave energy is scattered in all directions by diffraction process. If a long linear array of sensors facing the incident wave field is used to record the forward scatter and the back scatter is lost; naturally, some potentially useful information is also lost, in the sense that only a part of the object spectrum lying within a disc of radius equal to $\sqrt{2 k_{0}}$, where $k_{0}=2 \pi / \lambda$ and $\lambda$ is wavelength, is utilized. We propose to use a circular array of sensors (transreceivers) encircling the object so that both forward and backward scatter are captured. This results into doubling of the area of the spectrum coverage. Arbitrarily shaped measurement boundary was suggested by Porter [3] and Devaney and Beylkin [4] who have shown that on a straight line boundary or on a circular boundary it is sufficient to measure either the diffracted field or its normal derivative. A circular array for ultrasound holographic imaging was used by Quin et al. [5] but they have approximated a circular array by a series of linear arrays and then applied the back propagation algorithm [2]. A circular array of transceivers was also suggested for microwave diffraction tomography [6]-[7] where a near field diffraction phenomenon was used. The object Fourier transform was related to the scattered field through a two dimensional convolution relation. A circular array was also used by Otto et al. [8] for breast imaging. They have used back propagation technique for imaging and have also presented some experimental results.

In the present work we have extended the work of [4], [6], [7] to a large circular array (Radius $\approx 100 \lambda$ ). We establish a new result relating the scattered field to the object Fourier transform. This result, called here as Fourier Diffraction Theorem (FDT) for Circular Array, is as follows:

The scattered field measured with a large circular array surrounding the object is proportional to the Fourier transform of the object profile taken on the circumference a circle of radius equal to the wave number and centered at $\left(-k_{0} \cos \chi_{0},-k_{0} \sin \chi_{0}\right)$.

An important outcome of this result is that, since the measured field is itself the Fourier transform of the object profile, the need for

Manuscript received October 17, 1994; revised February 16, 1995. The work reported here was supported by Department of Ocean Development, Government of India, New Delhi.

The authors are with the Department of ECE, Indian Institute of Science, Bangalore 560012, India.

IEEE Log Number 9411936.

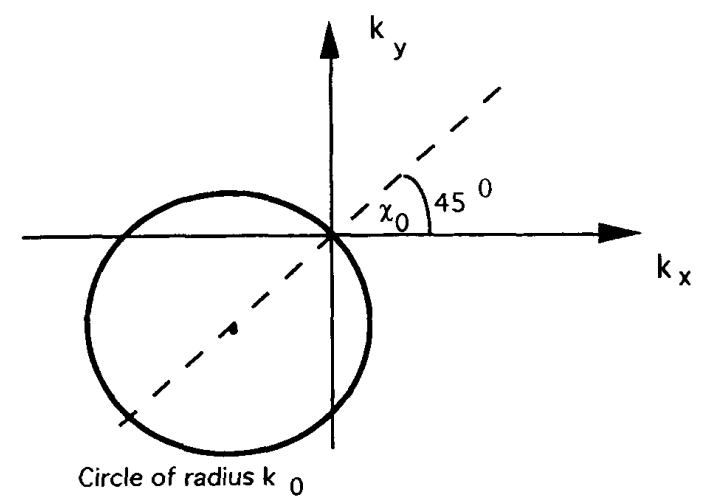

Fig. 1 For an angle of incidence, $c_{0}=45^{\circ}$, the scattered field received by a circular array is proportional to the object Fourier transform on a circle as shown.

computing the finite Fourier transform as in the case of linear array is overcome. Consequently, the effects of finite window such as the presence of sidelobes and a loss of resolution are no longer important.

\section{THEORY}

In order to be concise we shall not repeat the derivation for circular array given in [4], but start with (9) of [4]. The crucial step in our analysis is to replace the Hankel function by its large argument approximation $[9$, p. 364] and evaluate the summation with respect to $\mathrm{n}$. Using this approximation for Hankel function we get

$$
\begin{aligned}
k_{0}^{2} \bar{O}\left\{k-k_{0}\right\}= & -\frac{4 j}{2 \pi} \int_{-\pi}^{\pi} \psi_{s}\left(R, \sigma, \chi_{0}\right) \sqrt{\frac{\pi k_{0} R}{2}} \\
& \cdot e^{-j\left[k_{0} R-(\pi / 4)\right]} \sum_{n=-\infty}^{\infty} e^{j n(\chi-\sigma)} d \sigma .
\end{aligned}
$$

The summation in (1) can be shown to be

$$
\sum_{n=-\infty}^{\infty} e^{j n(\chi-\sigma)}=2 \pi \sum_{m=-\infty}^{\infty} \delta(\chi-\sigma-2 m \pi) .
$$

Note that in the interval of our interest, namely, $(-\pi, \pi)$, there is only one delta function at $\sigma=\chi$. Hence, in this interval (1) reduces to

$$
\begin{aligned}
& \tilde{O}\left\{k_{0}\left(\cos \chi-\cos \chi_{0}\right), k_{0}\left(\sin \chi-\sin \chi_{0}\right)\right\} \\
& =\frac{4}{k_{0}^{2}} e^{-j\left[k_{0} R+(\pi / 4)\right]} \sqrt{\frac{\pi k_{0} R}{2}} \psi_{s}\left(R, \chi, \chi_{0}\right) .
\end{aligned}
$$

The right hand side is simply the observed scattered field on a large circle. The left hand side is a Fourier transform of the object function which is evaluated on a circle of radius $k_{0}$ and centered at $k_{x}=-k_{0} \cos \chi_{0}$ and $k_{y}=-k_{0} \sin \chi_{0}$ (see Fig. 1). By changing the angle of incidence of the wavefront, $\chi_{0}$, it is possible to cover the entire Fourier plane with a series of circles. A disc of radius equal to $2 k_{0}$ is thus covered. This completes the proof of the FDT for a circular array.

\section{Verification of Fourier Diffraction TheOREM}

We shall now verify the FDT through an example where an exact scattered field as well as its object Fourier transform are known. Consider a liquid cylinder in water and assume that its refractive 


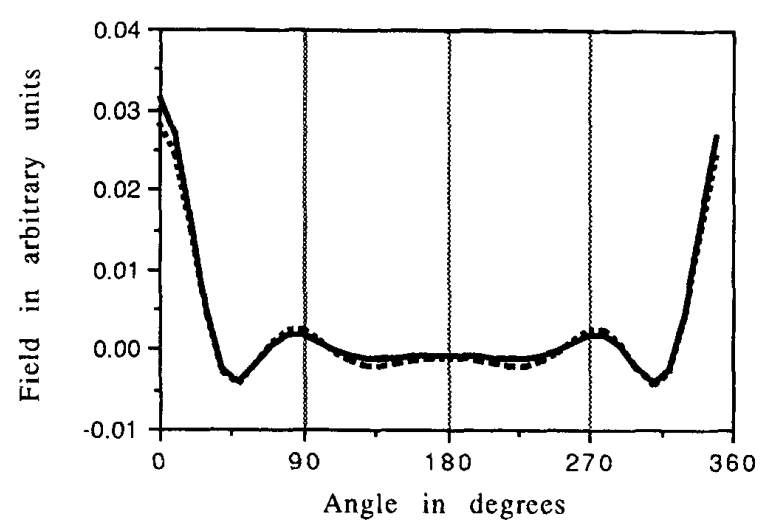

Fig. 2 A comparison of the scattered field due a liquid cylinder with the object Fourier transform. Dashed thick: scattered field and thin continuous line: object Fourier transform. For the liquid cylinder $\delta n=0.01, a=1.0 \lambda$.

index is slightly above that of the water, $\delta n \leq \lambda / 4 a$ where $\delta n$ is change in the refractive index, $a$ is radius of the cylinder and $\lambda$ is wavelength of illuminating wave (Born approximation). The scattered field due a liquid cylinder when it is illuminated by a plane wave was experimentally studied by Tamarkin [10] and theoretically by Bezuszka [11]. The expression given in [10] was programmed by us. Next, we evaluated the object profile Fourier transform

$$
\tilde{O}\left[k_{0}\left(\sin \chi-\sin \chi_{0}\right), k_{0}\left(\cos \chi-\cos \chi_{0}\right)\right]=2 \pi \delta_{n} a \frac{J_{1}(k a)}{k}
$$

where

$$
k=\sqrt{\left(\sin \chi-\sin \chi_{0}\right)^{2}+\left(\cos \chi-\cos \chi_{0}\right)^{2}} k_{0}
$$

as a function of $\chi$ for a fixed direction of illumination, in the present case, $\chi_{0}=0^{\circ}$. The scattered field measured by the circular array is now compared with the Fourier transform of the object profile evaluated on a circle centered at $\left(0,-k_{0}\right)$ in Fig. 2 . The mean square error was $1.759 \times 10^{-5}$.

\section{RECONSTRUCTION ALGORITHM}

For reconstruction we consider a circular array of 64 transceivers The angle of illumination, $\chi_{0}$, is varied over $360^{\circ}$ by switching on one sensor to transmit mode and keeping the remaining sensors in the receive mode. The received field can be expressed as a function of two parameters, namely, the angle of illumination and the angular coordinate of each sensor, that is, $\left(\chi, \chi_{0}\right)$. We must then map every point in space onto $\left(k_{x}, k_{y}\right)$ space. The reverse mapping, that is, from $\left(k_{x}, k_{y}\right)$ space onto $\left(\chi, \chi_{0}\right)$ space, is, however, more convenient to use. The to-and-from mapping functions are as follows:

$$
\cos \chi-\cos \chi_{0}=\frac{k_{x}}{k_{0}}, \quad \sin \chi-\sin \chi_{0}=\frac{k_{y}}{k_{0}} .
$$

Solving above equations we get the following inverse mapping functions:

$$
\begin{aligned}
& \chi_{0}=\tan ^{-1}\left\{\frac{-k_{y}-k_{x} \sqrt{\frac{2}{p}-1}}{-k_{x}+k_{y} \sqrt{\frac{2}{p}-1}}\right\} \\
& \chi=\tan ^{-1}\left\{\frac{k_{y}-k_{x} \sqrt{\frac{2}{p}-1}}{k_{x}+k_{y} \sqrt{\frac{2}{p}-1}}\right\}
\end{aligned}
$$

where

$$
p=\frac{k_{x}^{2}+k_{y}^{2}}{2 k_{0}^{2}} .
$$

Equations (3) and (4) together give a set of transformation equations which can be used to map $k$-plane into $\chi$-plane. Every point in the $k$-plane is mapped onto $\chi$-plane. The values of $\left(\chi, \chi_{0}\right)$ thus obtained may not correspond to any of those points where the scattered field is observed; then we must take recourse to some form of interpolation, for example, a bilinear interpolation,

$$
\tilde{O}\left(\chi, \chi_{0}\right)=\sum_{i} \sum_{j} \tilde{O}\left(\chi_{i}, \chi_{j}\right) h_{1}\left(\chi-\chi_{i}\right) h_{2}\left(\chi_{0}-\chi_{j}\right)
$$

where

$$
\begin{aligned}
h_{1}(\chi) & =1-\frac{|\chi|}{\Delta \chi} & & |\chi| \leq \Delta \chi \\
& =0 & & \text { otherwise }
\end{aligned} .
$$

and

$$
\begin{aligned}
h_{2}\left(\chi_{0}\right) & =1-\frac{\left|\chi_{0}\right|}{\Delta \chi_{0}} & & \left|\chi_{0}\right| \leq \Delta \chi_{0} \\
& =0 & & \text { otherwise }
\end{aligned}
$$

Here $\Delta \chi$ and $\Delta \chi_{0}$ are the sampling intervals. Once the values of Fourier obtained over a rectangular grid in $\left(k_{x}, k_{y}\right)$ space the inverse two dimensional Fourier transform can be easily computed to obtain the object profile. The above algorithm is essentially an adaptation of the frequency domain interpolation algorithm described in [2]. The algorithm was implemented on Microvax II and its performance is compared with the back propagation algorithm.

\section{Numerical Results}

Since a circular array captures the entire diffracted energy, that is, both forward and backward scattered energy, a greater part of the object spectrum is utilized, indeed twice that of forward-scatter-only (linear array) set up. Consequently, we expect a better resolution of small inhomogeneities. To demonstrate this we have carried out the following numerical experiment. Two concentric cylinders of radii $0.4 \lambda$ and $.80 \lambda$, having refractive index contrasts with respect to the surrounding medium as 0.015 and 0.005 , were insonified with a radiation of wavelength $1.0 \mathrm{\lambda}$. A circular array of 64 transceivers is assumed. For comparison we have also considered a linear array of the same length and one transmitter located on the broad side and the scattered field was calculated using the object Fourier transform over semi circular arcs as in [2]. For circular array, however, the scattered field was computed using the exact solution given in [10]-[11]. The reconstruction (a central slice) of the target is shown in Fig. 3. The reconstruction obtained using a linear array is shown in Fig. 3(a) and that obtained using a circular array is shown in Fig. 3(b). Clearly the circular array outperforms the equivalent linear array in terms of its improved resolution made possible by the larger coverage of the disc in Fourier space (see Fig. 1).

Next, we like to underline the role of the array size on object reconstruction. When using a linear array it is necessary that the array output has to be Fourier transformed before it is used for reconstruction. Consequently, the errors in the Fourier transformation due to finite size of the array will degrade the reconstruction. This effect is demonstrated in Fig. 4. The first three figures (4(a)-4(c)) were obtained using a linear array of three different sizes, namely, 64, 128 , and 512 sensors spaced at $\lambda / 2$. The scattered field was computed 


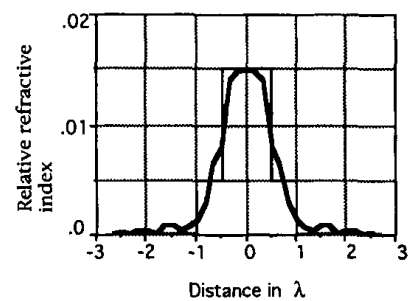

(a)

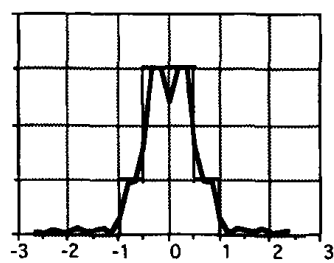

Distance in $\lambda$

(b)
Fig. 3 A comparison of reconstructions obtained using (a) linear array and (b) circular array. The object is made of two concentric cylinders. Thick line: reconstruction and thin line: actual cross section (see text for details).
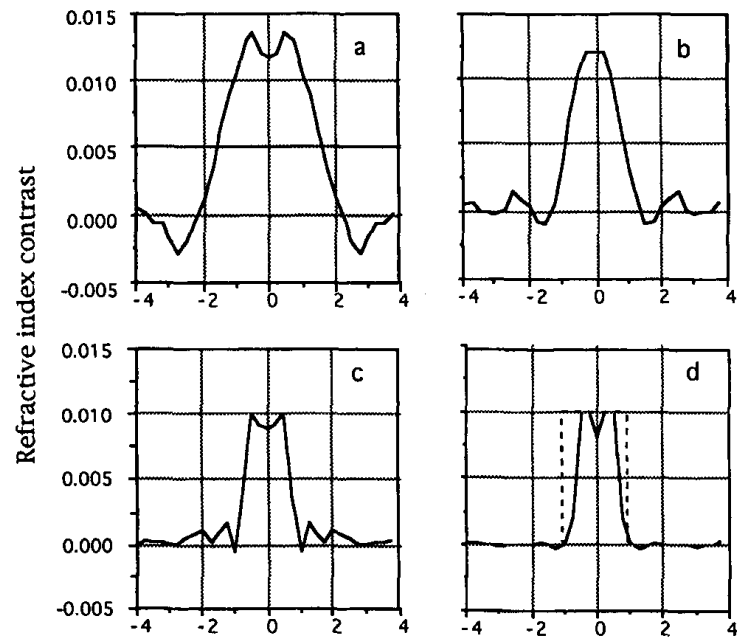

Distance in $\lambda$

Fig. 4 Reconstructions with linear array of different lengths (a) 64 sensors (b) 128 sensors and (c) 512 sensors. For comparison the reconstruction with circular array of 64 sensors is shown in (d).

using the exact solution given in [10]-[11]. The reconstruction in Fig. 4(c) was obtained using a 64 element circular array (rad $=100 \lambda)$. The sensor spacing now need not be $\lambda / 2$, as we do not compute the spatial Fourier transform. The scattered field was computed once again using the exact solution given in [10]-[11]. The number of illuminations taken for this study was 64 . The reconstruction obtained with a circular array is far superior to that obtained with a linear array of much larger size.

\section{CONCLUSION}

In conclusion, it is shown that a circular array for diffraction tomographic imaging has some important advantages. Apart from not having to physically go around the object, a circular array yields better resolution on account of larger bandwidth used for reconstruction. Further, since the scattered field received by the circular array is proportional to the object Fourier transform, the reconstruction is not affected by the errors induced by finite array size.

\section{ACKNOWLEDGMENT}

The authors are grateful to anonymous reviewers who brought to our attention some of the recent work with some bearing on the present paper.

\section{REFERENCES}

[1] A. J. Devaney, "Diffraction tomography," in Inverse Methods in Electromagnetic Imaging Part 2, W. M. Boerner et al., Eds. New York: D. Reidel, 1985, pp. 1107-1135.

[2] A. C. Kak, "Tomographic imaging with diffracting and nondiffracting sources," in Array Signal Processing, S. Hayakin, Ed. Englewood Cliffs, NJ: Prentice-Hall, 1985, pp. 351-428.

[3] R. Porter, "Determination of structure of weak scatterers from holographic images," Opt. Commun., vol. 39, pp. 362-364, 1981.

[4] A. J. Devaney and G. Beylkin, "Diffraction tomography using arbitrary transmitter and receiver surfaces," Ultrason. Imag., vol. 6, pp. 181-193, 1984.

[5] Z. Qin et al., "Circular array ultrasound holographic imaging using the linear array approach," IEEE Trans.Ultrason., Ferroelect., Freq. Cont., vol. 36, p. $485,1989$.

[6] J. M. Ruis, M. Ferrando, L. Jofre, E. De Los Reyes, A. Elias, and A. Broquet, "Microwave tomography: An algorithm for cylindrical geometries," Electron. Lett., May 21, 1987, vol. 23, pp. 564-565.

[7] J. M. Ruis, C. Pichot, L. Jofre, J. C. Bolomy, N. Joachimowicz, A. Broquet, and M. Ferrando, "Planar and cylindrical active microwave temperature imaging: Numerical simulations," IEEE. Trans. Med. Imag., vol. 11, pp. $457-469,1992$.

[8] G. P. Otto, P. J. Martin, M. P. Andre, B. A. Spivey, T. Barrett, and D. A. Palmer, "Phantom studies of phase aberration correction methods in diffraction tomography," Medical Physics, vol. 21, p. 998, 1994.

[9] M. Abramowitz and I. A. Stegun, Handbook of Mathematical Functions. New York: NBS, 1964.

[10] P. Tamarkin, "Scattering of an underwater ultrasonic beam from liquid cylindrical obstacles," J. Acoust. Soc. Amer., vol. 21, pp. 612-616, 1949.

[11] S. J. Bezuszka, "Scattering of underwater plane ultrasonic waves by liquid cylindrical obstacles," J. Acoust. Soc. Amer., vol. 25, pp. 1090-1095, 1953.

\section{Analytical and Numerical Treatment of Pulsed Wave Propagation into a Viscous Fluid}

Reinhold Ludwig, Senior Member, IEEE, and Peter L. Levin

Abstract-A transient analytical expression for the propagation of pulsed ultrasound through a viscous fluid is derived by evaluating the Laplace transform in the complex domain. The numerical solution of the viscous wave equation without any restricting approximations is developed for a full space with an impulsive excitation at the origin Different values of the viscosity coefficient for a given sound speed clearly delineate the transition from a pure parabolic, or diffusive to a pure hyperbolic or wave propagation behavior. This region is often of crucial importance from a practical point of view as ultrasonic instrumentation in medical imaging and nondestructive testing must compensate not only for phase differences due to propagation delays but also for pulse distortion due to attenuation mechanisms in the medium of interest.

Manuscript received November 8, 1994; revised March 8, 1995.

The authors are with the Department of Electrical and Computer Engineering, Worcester Polytechnic Institute, Worcester, MA 01609 USA. IEEE Log Number 9411934 